\title{
ON THE ALGEBRA OF SMOOTH OPERATORS
}

\author{
TOMASZ CiAŚ
}

\begin{abstract}
Let $s$ be the space of rapidly decreasing sequences. We give the spectral representation of normal elements in the Fréchet algebra $L\left(s^{\prime}, s\right)$ of the so-called smooth operators. We also characterize closed commutative *-subalgebras of $L\left(s^{\prime}, s\right)$ and establish a Hölder continuous functional calculus in this algebra. The key tool is the property $(D N)$ of $s$.
\end{abstract}

\section{Introduction}

The space $s$ of rapidly decreasing sequences plays significant role in the structure theory of nuclear Fréchet spaces. One of the most explicit example of this is provided by the KōmuraKōmura theorem which implies that a Fréchet space is nuclear if and only if it is isomorphic to some closed subspace of $s^{\mathbb{N}}$ (see e.g. [11, Cor. 29.9]). The space $s$ has also many interesting representations. For instance, it is isomorphic as a Fréchet space to the Schwartz space $\mathcal{S}\left(\mathbb{R}^{n}\right)$ of rapidly decreasing smooth functions, the space $\mathcal{D}(K)$ of test functions with support in a compact set $K \subset \mathbb{R}^{n}$ such that $\operatorname{int}(K) \neq \emptyset$, the space $C^{\infty}(M)$ of smooth functions on a compact smooth manifold $M$, the space $C^{\infty}[0,1]$ of smooth functions on the interval $[0,1]$. Finally, the space $s$ and all of the spaces above are Fréchet commutative algebras with the pointwise multiplication. However, these algebras do not have to be isomorphic as algebras (for instance, $s$ and $C^{\infty}[0,1]$ with the pointwise multiplication are not isomorphic as algebras).

A natural candidate for "the noncommutative $s$ " is the algebra $L\left(s^{\prime}, s\right)$ of the so-called smooth operators, where multiplication is just the composition of operators (let us note that $s \subseteq s^{\prime}$ continuously). It appears in $K$-theory for Fréchet algebras ([12, Def. 2.1], [1, Ex. 2.12], [9]) and in $C^{*}$-dynamical systems $([7, \mathrm{Ex} .2 .6])$. The algebra $L\left(s^{\prime}, s\right)$ is also an example of a dense smooth subalgebra of a $C^{*}$-algebra (precisely, $L\left(s^{\prime}, s\right)$ is a dense subalgebra of the $C^{*}$ algebra $K\left(l_{2}\right)$ of compact operators on $l_{2}$ ) which is especially important in the noncommutative geometry (see [1], 2], 3, p. 23, 183-184]). From the philosophical point of view $C^{*}$-algebras just corespond to analogues of topological spaces whereas some of their dense smooth subalgebras play the role of smooth structures.

Representations of $s$ may lead up to representations of the algebra $L\left(s^{\prime}, s\right)$. Many of them are collected in [6. Th. 2.1]. For example, $L\left(s^{\prime}, s\right)$ is isomorphic as a Fréchet ${ }^{*}$-algebra to the following *-algebras of continuous linear operators with the appropriate multiplication and involution: $L\left(\mathcal{S}^{\prime}\left(\mathbb{R}^{n}\right), \mathcal{S}\left(\mathbb{R}^{n}\right)\right), L\left(\mathcal{E}^{\prime}(M), C^{\infty}(M)\right), L\left(\mathcal{E}^{\prime}[0,1], C^{\infty}[0,1]\right)$, where $\mathcal{S}^{\prime}\left(\mathbb{R}^{n}\right)$ is the space of tempered distributions, $M \subset \mathbb{R}^{n}$ is a compact smooth manifold, $\mathcal{E}^{\prime}(M)$ is the space of distributions on $M$ and $\mathcal{E}^{\prime}[0,1]$ is the space of distributions with the support in [0,1]. It is also worth

\footnotetext{
${ }^{1}$ 2010 Mathematics Subject Classification. Primary: 46H35, 46J25, 46H30. Secondary: 46H15, 46K10, 46A11, $46 \mathrm{~L} 05$.

Key words and phrases: Topological algebras of operators, topological algebras with involution, representations of commutative topological algebras, functional calculus in topological algebras, nuclear Fréchet spaces, $C^{*}$ algebras, smooth operators, space of rapidly decreasing smooth functions.
} 
to mention two extra representations of $L\left(s^{\prime}, s\right)$ : the algebra of rapidly decreasing matrices

$$
\mathcal{K}:=\left\{\left(\xi_{j, k}\right)_{j, k \in \mathbb{N}}: \sup _{j, k \in \mathbb{N}}\left|\xi_{j, k}\right| j^{q} k^{q}<\infty \text { for all } q \in \mathbb{N}_{0}\right\}
$$

with matrix multiplication and conjugation of the transpose as an involution (see e.g. [3, p. 238], [12, Def. 2.1]), and also the algebra $\mathcal{S}\left(\mathbb{R}^{2}\right)$ equipped with the Volterra convolution $(f \cdot g)(x, y):=$ $\int_{\mathbb{R}} f(x, z) g(z, y) \mathrm{d} z$ and the involution $f^{*}(x, y):=\overline{f(y, x)}$ (see e.g. [1, Ex. 2.12]).

The purpose of this paper is to present some spectral, algebra and functional calculus properties of the algebra of smooth operators. The results are derived from the basic theory of nuclear Fréchet spaces and the theory of bounded operators on a separable Hilbert space. The heart of the paper is the theorem on the spectral representation of normal elements in $L\left(s^{\prime}, s\right)$ (Theorem 3.1). In the proof we use the fact that the operator norm $\|\cdot\|_{l_{2} \rightarrow l_{2}}$ is a dominating norm on $L\left(s^{\prime}, s\right)$ (Proposition 3.2). As a by-product we obtain a kind of spectral description of normal elements of $L\left(s^{\prime}, s\right)$ among those of $K\left(l_{2}\right)$ (Corollary 3.6). Next, we characterize closed commutative ${ }^{*}$-subalgebras of $L\left(s^{\prime}, s\right)$. We prove that every such a subalgebra is generated by a single operator and also by its spectral projections (Theorem 4.8), and, moreover, that it is a Köthe algebra with pointwise multiplication. To do this, we show that every closed commutative *-subalgebra of $L\left(s^{\prime}, s\right)$ has some canonical Schauder basis (Lemma 4.4). Finally, we establish a Hölder-continuous functional calculus in $L\left(s^{\prime}, s\right)$ (Corollary [5.1) and we prove the functional calculus theorem for normal elements in this algebra (Theorem 5.2).

By a Fréchet space we mean a complete metrizable locally convex space. A Fréchet algebra is a Fréchet space which is an algebra with a continuous multiplication. A Fréchet ${ }^{*}$-algebra is a Fréchet algebra with an involution.

We use the standard notation and terminology. All the notions from Functional Analysis are explained in [11] and those from topological algebras in [8] or [17].

\section{Preliminaries}

Throughout the paper, $\mathbb{N}$ will denote the set of natural numbers $\{1,2, \ldots\}$ and $\mathbb{N}_{0}:=\mathbb{N} \cup\{0\}$.

By projection on $l_{2}$ we always mean a continuous orthogonal (self-adjoint) projection.

We define the space of rapidly decreasing sequences as a Fréchet space

$$
s:=\left\{\xi=\left(\xi_{j}\right)_{j \in \mathbb{N}} \in \mathbb{C}^{\mathbb{N}}:|\xi|_{q}:=\left(\sum_{j=1}^{\infty}\left|\xi_{j}\right|^{2} j^{2 q}\right)^{\frac{1}{2}}<\infty \text { for all } q \in \mathbb{N}_{0}\right\}
$$

with the topology corresponding to the system $\left(|\cdot|_{q}\right)_{q \in \mathbb{N}_{0}}$ of norms. Its strong dual is isomorphic to the space of slowly increasing sequences

$$
s^{\prime}:=\left\{\xi=\left(\xi_{j}\right)_{j \in \mathbb{N}} \in \mathbb{C}^{\mathbb{N}}:|\xi|_{q}^{\prime}:=\left(\sum_{j=1}^{\infty}\left|\xi_{j}\right|^{2} j^{-2 q}\right)^{\frac{1}{2}}<\infty \text { for some } q \in \mathbb{N}_{0}\right\}
$$

equipped with the inductive limit topology given by the system $\left(|\cdot|_{q}^{\prime}\right)_{q \in \mathbb{N}_{0}}$ of norms.

Every $\eta \in s^{\prime}$ corresponds to the continuous functional $\xi \mapsto\langle\xi, \eta\rangle$ on $s$, where

$$
\langle\xi, \eta\rangle:=\sum_{j=1}^{\infty} \xi_{j} \overline{\eta_{j}}
$$


Futhermore, by the Cauchy-Schwartz inequality we get

$$
|\langle\xi, \eta\rangle| \leq|\xi|_{q}|\eta|_{q}^{\prime}
$$

for all $q \in \mathbb{N}_{0}, \xi \in s$ and $\eta \in s^{\prime}$ with $|\eta|_{q}^{\prime}<\infty$.

For $1 \leq p<\infty$ and the Köthe matrix $\left(a_{j, q}\right)_{j \in \mathbb{N}, q \in \mathbb{N}_{0}}$ we define the Köthe space

$$
\lambda^{p}\left(a_{j, q}\right):=\left\{\xi=\left(\xi_{j}\right)_{j \in \mathbb{N}} \in \mathbb{C}^{\mathbb{N}}:|\xi|_{p, q}:=\left(\sum_{j=1}^{\infty}\left|\xi_{j} a_{j, q}\right|^{p}\right)^{\frac{1}{p}}<\infty \text { for all } q \in \mathbb{N}_{0}\right\}
$$

and for $p=\infty$

$$
\lambda^{\infty}\left(a_{j, q}\right):=\left\{\xi=\left(\xi_{j}\right)_{j \in \mathbb{N}} \in \mathbb{C}^{\mathbb{N}}:|\xi|_{\infty, q}:=\sup _{j \in \mathbb{N}}\left|\xi_{j}\right| a_{j, q}<\infty \text { for all } q \in \mathbb{N}_{0}\right\}
$$

with the topology generated by norms $\left(|\cdot|_{p, q}\right)_{q \in \mathbb{N}_{0}}$ (see e.g. [11, Def. on p. 326]). Please note that sometimes these spaces are Fréchet ${ }^{*}$-algebras with the pointwise multiplication.

Now, the space $s$ is just the Köthe space $\lambda^{2}\left(j^{q}\right)$. Moreover, since the space $s$ is a nuclear Fréchet space, it is isomorphic to any Köthe space $\lambda^{p}\left(j^{q}\right)$ for $1 \leq p \leq \infty$ (see e.g. [11, Prop. 28.16, Ex. $29.4(1)]$ ). We use $l_{2}$-norms to simplify futher computations, for example we have $|\cdot|_{0}=|\cdot|_{0}^{\prime}=\|\cdot\|_{l_{2}}$.

It is well known that the space $L\left(s^{\prime}, s\right)$ of continuous linear operators from $s^{\prime}$ to $s$ with the fundamental system of norms $\left(\|\cdot\|_{q}\right)_{q \in \mathbb{N}_{0}}$,

$$
\|x\|_{q}:=\sup _{|\xi|_{q}^{\prime} \leq 1}|x \xi|_{q}
$$

is isomorphic to $s$ as Fréchet space. Moreover, $L\left(s^{\prime}, s\right)$ is isomorphic to $s \widehat{\otimes} s$, the completed tensor product of $s$ (see [10, $\S 41.7(5)]$ ).

Since the canonical inclusion $j: s \hookrightarrow s^{\prime}$ is continuous, thus for $x, y \in L\left(s^{\prime}, s\right)$

$$
x \cdot y:=x \circ j \circ y
$$

is in $L\left(s^{\prime}, s\right)$ as well and with this operation $L\left(s^{\prime}, s\right)$ is a Fréchet algebra.

The diagram

$$
l_{2} \hookrightarrow s^{\prime} \rightarrow s \hookrightarrow l_{2}
$$

defines the canonical (continuous) embeding of the algebra $L\left(s^{\prime}, s\right)$ into the algebra $L\left(l_{2}\right)$ of continuous linear operators on the Hilbert space $l_{2}$. In fact, this inclusion acts into the space $K\left(l_{2}\right)$ of compact operators on $l_{2}$ and the sequence of singular numbers of elements in $L\left(s^{\prime}, s\right)$ belongs to $s$ (see [6. Prop. 3.1, Cor. 3.2]). Therefore, $L\left(s^{\prime}, s\right)$ can be regarded as some class of compact operators on $l_{2}$. Clearly, the multiplication in $L\left(s^{\prime}, s\right)$ coincides with the composition in $L\left(l_{2}\right)$, and further, $L\left(s^{\prime}, s\right)$ is invariant under the hilbertian involution $x \mapsto x^{*}$.

To see this, let us consider the Fréchet ${ }^{*}$-algebra of rapidly decreasing matrices

$$
\mathcal{K}:=\left\{\Xi=\left(\xi_{j, k}\right)_{j, k \in \mathbb{N}}:\||\Xi|\|_{q}:=\sup _{j, k \in \mathbb{N}}\left|\xi_{j, k}\right| j^{q} k^{q}<\infty \text { for all } q \in \mathbb{N}_{0}\right\}
$$

with the matrix multiplication, the involution defined by $\left(\left(\xi_{j, k}\right)_{j, k \in \mathbb{N}}\right)^{*}:=\left(\overline{\xi_{k, j}}\right)_{j, k \in \mathbb{N}}$ and with $\left(\left|\|\cdot \mid\|_{q}\right)_{q \in \mathbb{N}_{0}}\right.$ as its fundamental sequence of norms. By [6, Th. 2.1], $\Phi: L\left(s^{\prime}, s\right) \rightarrow \mathcal{K}, \Phi(x):=$ $\left(\left\langle x e_{k}, e_{j}\right\rangle\right)_{j, k \in \mathbb{N}}$ is the algebra isomorphism and we have

$$
\Phi(x)^{*}=\left(\overline{\left\langle x e_{j}, e_{k}\right\rangle}\right)_{j, k \in \mathbb{N}}=\left(\left\langle x^{*} e_{k}, e_{j}\right\rangle\right)_{j, k \in \mathbb{N}} .
$$


Hence, $x^{*}=\Phi^{-1}\left(\Phi(x)^{*}\right) \in L\left(s^{\prime}, s\right)$ and $\Phi$ is even a ${ }^{*}$-isomorphism. Clearly, for every matrix $\Xi \in \mathcal{K}$ and $q \in \mathbb{N}_{0},\|\| \Xi^{*} \mid\left\|_{q}=\right\|\|\Xi\|_{q}$, thus the hilbertian involution is continuous on $L\left(s^{\prime}, s\right)$.

The Fréchet algebra $L\left(s^{\prime}, s\right)$ with the involution * is called the algebra of smooth operators. We will also consider the algebra with unit

$$
\widetilde{L\left(s^{\prime}, s\right)}:=\left\{x+\lambda \mathbf{1}: x \in L\left(s^{\prime}, s\right), \lambda \in \mathbb{C}\right\},
$$

where 1 is the identity operator on $l_{2}$. We endow the algebra $\widetilde{L\left(s^{\prime}, s\right)}$ with the product topology.

Now, we shall recall some basic spectral properties of the algebra $L\left(s^{\prime}, s\right)$. For the sake of convenience, we state the following definition.

Definition 2.1. We say that a sequence $\left(\lambda_{n}\right)_{n \in \mathbb{N}} \subset \mathbb{C}$ is a sequence of eigenvalues of an infinite dimensional compact operator $x$ on $l_{2}$ if it satisfies the following conditions:

(i) $\left\{\lambda_{n}\right\}_{n \in \mathbb{N}}$ is the set of eigenvalues of $x$ without zero;

(ii) $\left|\lambda_{1}\right| \geq\left|\lambda_{2}\right| \geq \ldots>0$ and if two eigenvalues has the same absolute value then we can ordered them in an arbitrary way;

(iii) the number of occurrences of the eigenvalue $\lambda_{n}$ is equal to its geometric multiplicity (i.e., the dimension of the space $\left.\operatorname{ker}\left(\lambda_{n} \mathbf{1}-x\right)\right)$.

Proposition 2.3 is well known (see e.g. [9]) and it is a simple consequence of Proposition 2.2. Proofs of Propositions 2.2, 2.4 one can find in [6, Th. 3.3, Cor. 3.5].

Proposition 2.2. An operator in $\widetilde{L\left(s^{\prime}, s\right)}$ is invertible if and only if it is invertible in $L\left(l_{2}\right)$.

Proposition 2.3. The algebra $\widetilde{L\left(s^{\prime}, s\right)}$ is a Q-algebra, i.e., the set of invertible elements is open.

Proposition 2.4. The spectrum of $x$ in $L\left(s^{\prime}, s\right)$ equals the spectrum of $x$ in $L\left(l_{2}\right)$ and it consists of zero and the set of eigenvalues. If moreover, $x$ is infinite dimensional, then the sequence of eigenvalues of $x$ (see Definition 2.1) belongs to $s$.

The first part of the following proposition is also known (see e.g. [12, Lemma 2.2]). We give a simple proof that norms $\|\cdot\|_{q}$ are submultiplicative.

Proposition 2.5. The algebra $L\left(s^{\prime}, s\right)$ is $m$-locally convex, i.e., it has a fundamental system of submultiplicative norms. Moreover, $\|x y\|_{q} \leq\|x\|_{q}\|y\|_{q}$ for every $q \in \mathbb{N}_{0}$.

Proof. Let $x, y \in L\left(s^{\prime}, s\right)$ and let $B_{q}, B_{q}^{\prime}$ denote the closed unit ball for the norms $|\cdot|_{q},|\cdot|_{q}^{\prime}$, respectively. Clearly, $y\left(B_{q}^{\prime}\right) \subseteq\|y\|_{q} B_{q}$ and $B_{q} \subseteq B_{q}^{\prime}$. Hence

$$
\begin{aligned}
\|x y\|_{q} & =\sup _{|\xi|_{q}^{\prime} \leq 1}|x(y(\xi))|_{q}=\sup _{\eta \in y\left(B_{q}^{\prime}\right)}|x(\eta)|_{q} \leq \sup _{\eta \in\|y\|_{q} B_{q}}|x(\eta)|_{q}=\|y\|_{q} \sup _{\eta \in B_{q}}|x(\eta)|_{q} \\
& \leq\|y\|_{q} \sup _{\eta \in B_{q}^{\prime}}|x(\eta)|_{q}=\|x\|\left\|_{q}\right\| y \|_{q} .
\end{aligned}
$$




\section{Spectral representation of normal elements}

In this section we prove the following theorem on the spectral representation of normal elements in $L\left(s^{\prime}, s\right)$.

Theorem 3.1. Every infinite dimensional normal operator $x$ in $L\left(s^{\prime}, s\right)$ has the unique spectral representation $x=\sum_{n=1}^{\infty} \lambda_{n} P_{n}$, where $\left(\lambda_{n}\right)_{n \in \mathbb{N}}$ is a decreasing (according to the modulus) sequence in $s$ of nonzero pairwise different elements, $\left(P_{n}\right)_{n \in \mathbb{N}}$ is a sequence of nonzero pairwise orthogonal finite dimensional projections belonging to $L\left(s^{\prime}, s\right)$ (i.e., the canonical inclusion of $P_{n}$ into $L\left(l_{2}\right)$ is a projection on $\left.l_{2}\right)$ and the series converges absolutely in $L\left(s^{\prime}, s\right)$. Moreover, $\left(\left|\lambda_{n}\right|^{\theta}|| P_{n} \|_{q}\right)_{n \in \mathbb{N}} \in s$ for all $q \in \mathbb{N}_{0}$ and all $\theta \in(0,1]$.

Before we prove this result, we first need to do some preparation. Let us recall (see [11, Def. on p. 359 and Lemma 29.10]) that a Fréchet space $\left(X,\left(\|\cdot\|_{q}\right)_{q \in \mathbb{N}_{0}}\right)$ has the property $(D N)$ if there is a continuous norm $\|\cdot\|$ on $X$ such that for any $q \in \mathbb{N}_{0}$ and $\theta \in(0,1)$ there is $r \in \mathbb{N}_{0}$ and $C>0$ such that for all $x \in X$

$$
\|x\|_{q} \leq C\|x\|^{1-\theta}\|x\|_{r}^{\theta} .
$$

The norm $\|\cdot\|$ is called a dominating norm.

The following result is essentially due to K. Piszczek (see [13, Th. 4]).

Proposition 3.2. The norm $\|\cdot\|_{l_{2} \rightarrow l_{2}}$ is a dominating norm on $L\left(s^{\prime}, s\right)$.

Proof. Clearly, $\|\cdot\|_{l_{2} \rightarrow l_{2}}=\|\cdot\|_{0}$. By [16, Th. 4.3] (see the proof), the conclusion is equivalent to the following condition

$$
\forall q \in \mathbb{N}_{0} \forall \theta>0 \exists r \in \mathbb{N}_{0} \exists C>0 \forall h>0 \forall x \in L\left(s^{\prime}, s\right) \quad\|x\|_{q} \leq C\left(h^{\theta}\|x\|_{r}+\frac{1}{h}\|x\|_{0}\right) .
$$

From Hölder's inequality, the norm $|\cdot|_{0}$ is a dominating norm on $s$, hence again by [16, Th. 4.3] as above we get

$$
\forall q \in \mathbb{N}_{0} \forall \eta>0 \exists r \in \mathbb{N}_{0} \exists D_{0}>0 \forall k>0 \forall \xi \in s \quad|\xi|_{q} \leq D_{0}\left(k^{\eta}|\xi|_{r}+\frac{1}{k}|\xi|_{0}\right) .
$$

Now, by the bipolar theorem (see e.g. [11, Th. 22.13]) we obtain (follow the proof of [11, Lemma 29.13]) an equivalent condition

$$
\forall q \in \mathbb{N}_{0} \forall \eta>0 \exists r \in \mathbb{N}_{0} \exists D>0 \forall k>0 \quad U_{q}^{\circ} \subset D\left(k^{\eta} U_{r}^{\circ}+\frac{1}{k} U_{0}^{\circ}\right),
$$

where $U_{q}:=\left\{\xi \in s:|\xi|_{q} \leq 1\right\}$ and $U_{q}^{\circ}$ is its polar. If $\theta>0$ and $h \in(0,1]$ are given, we define $\eta:=2 \theta+1, k:=\sqrt{h}$. Since $k^{2 \eta} \leq k^{\eta-1}$, we obtain

$$
\begin{aligned}
U_{q}^{\circ} \otimes U_{q}^{\circ} & :=\left\{x \otimes y: x, y \in U_{q}^{\circ}\right\} \subset D\left(k^{\eta} U_{r}^{\circ}+\frac{1}{k} U_{0}^{\circ}\right) \otimes D\left(k^{\eta} U_{r}^{\circ}+\frac{1}{k} U_{0}^{\circ}\right) \\
& \subset D^{2}\left(k^{2 \eta} U_{r}^{\circ} \otimes U_{r}^{\circ}+2 k^{\eta-1} U_{r}^{\circ} \otimes U_{r}^{\circ}+\frac{1}{k^{2}} U_{0}^{\circ} \otimes U_{0}^{\circ}\right) \\
& \subset 3 D^{2}\left(k^{\eta-1} U_{r}^{\circ} \otimes U_{r}^{\circ}+\frac{1}{k^{2}} U_{0}^{\circ} \otimes U_{0}^{\circ}\right)=3 D^{2}\left(h^{\theta} U_{r}^{\circ} \otimes U_{r}^{\circ}+\frac{1}{h} U_{0}^{\circ} \otimes U_{0}^{\circ}\right) .
\end{aligned}
$$


Since $r$ and $D$ in condition (11) can be choosen so that $q \leq r$ and $D \geq 1$, thus for $h>1$ it holds

$$
U_{q}^{\circ} \otimes U_{q}^{\circ} \subset U_{r}^{\circ} \otimes U_{r}^{\circ} \subset 3 D^{2}\left(h^{\theta} U_{r}^{\circ} \otimes U_{r}^{\circ}+\frac{1}{h} U_{0}^{\circ} \otimes U_{0}^{\circ}\right),
$$

hence we have shown

$$
\forall q \in \mathbb{N}_{0} \forall \theta>0 \exists r \in \mathbb{N}_{0} \exists C>0 \forall h>0 \quad U_{q}^{\circ} \otimes U_{q}^{\circ} \subset C\left(h^{\theta} U_{r}^{\circ} \otimes U_{r}^{\circ}+\frac{1}{h} U_{0}^{\circ} \otimes U_{0}^{\circ}\right) .
$$

Therefore, for all $x:=\sum_{j=1}^{n} x_{j} \otimes y_{j} \in s \otimes s$ we get

$$
\begin{aligned}
\sup \left\{|z(x)|: z \in U_{q}^{\circ} \otimes U_{q}^{\circ}\right\} & \leq C \sup \left\{|z(x)|: z \in h^{\theta} U_{r}^{\circ} \otimes U_{r}^{\circ}+\frac{1}{h} U_{0}^{\circ} \otimes U_{0}^{\circ}\right\} \\
& =C \sup \left\{\left|\left(z^{\prime}+z^{\prime \prime}\right)(x)\right|: z^{\prime} \in h^{\theta} U_{r}^{\circ} \otimes U_{r}^{\circ}, z^{\prime \prime} \in \frac{1}{h} U_{0}^{\circ} \otimes U_{0}^{\circ}\right\} \\
& \leq C \sup \left\{\left|z^{\prime}(x)\right|+\left|z^{\prime \prime}(x)\right|: z^{\prime} \in h^{\theta} U_{r}^{\circ} \otimes U_{r}^{\circ}, z^{\prime \prime} \in \frac{1}{h} U_{0}^{\circ} \otimes U_{0}^{\circ}\right\} \\
& =C\left(h^{\theta} \sup \left\{|z(x)|: z \in U_{r}^{\circ} \otimes U_{r}^{\circ}\right\}+\frac{1}{h} \sup \left\{|z(x)|: z \in U_{0}^{\circ} \otimes U_{0}^{\circ}\right\}\right) .
\end{aligned}
$$

Let $\chi: s \otimes s \rightarrow L\left(s^{\prime}, s\right), \chi\left(\sum_{j=1}^{n} x_{j} \otimes y_{j}\right)(z):=\sum_{j=1}^{n} z\left(y_{j}\right) x_{j}$. We have for all $p \in \mathbb{N}_{0}$

$$
\begin{aligned}
\sup \left\{\left|z\left(\sum_{j=1}^{n} x_{j} \otimes y_{j}\right)\right|: z \in U_{p}^{\circ} \otimes U_{p}^{\circ}\right\} & =\sup \left\{\left|\sum_{j=1}^{n} z_{1}\left(x_{j}\right) z_{2}\left(y_{j}\right)\right|: z_{1}, z_{2} \in U_{p}^{\circ}\right\} \\
& =\sup \left\{\left|z_{1}\left(\sum_{j=1}^{n} z_{2}\left(y_{j}\right) x_{j}\right)\right|: z_{1}, z_{2} \in U_{p}^{\circ}\right\} \\
& =\sup \left\{\left|\sum_{j=1}^{n} z\left(y_{j}\right) x_{j}\right|_{p}: z \in U_{p}^{\circ}\right\} \\
& =\sup \left\{\left|\chi\left(\sum_{j=1}^{n} x_{j} \otimes y_{j}\right)(z)\right|_{p}: z \in U_{p}^{\circ}\right\} \\
& =\left\|\chi\left(\sum_{j=1}^{n} x_{j} \otimes y_{j}\right)\right\|_{p}
\end{aligned}
$$

Hence

$$
\left\|\chi\left(\sum_{j=1}^{n} x_{j} \otimes y_{j}\right)\right\|_{q} \leq C\left(h^{\theta}\left\|\chi\left(\sum_{j=1}^{n} x_{j} \otimes y_{j}\right)\right\|_{r}+\frac{1}{h}\left\|\chi\left(\sum_{j=1}^{n} x_{j} \otimes y_{j}\right)\right\|_{0}\right) .
$$

Finally, since the set $\left\{\chi\left(\sum_{j=1}^{n} x_{j} \otimes y_{j}\right): x_{j}, y_{j} \in s, n \in \mathbb{N}\right\}$ is dense in $L\left(s^{\prime}, s\right)$, thus

$$
\|x\|_{q} \leq C\left(h^{\theta}\|x\|_{r}+\frac{1}{h}\|x\|_{0}\right)
$$

for all $x \in L\left(s^{\prime}, s\right)$.

Lemma 3.3. Let $\left(E,\left(\|\cdot\|_{q}\right)_{q \in \mathbb{N}_{0}}\right)$ be a Fréchet space with the property $(D N)$ and let $\|\cdot\|_{p}$ be a dominating norm. If $\left(x_{n}\right)_{n \in \mathbb{N}} \subset E,\left(\lambda_{n}\right)_{n \in \mathbb{N}} \subset \mathbb{C}$ satisfy conditions 
(i) $\sup _{n \in \mathbb{N}}\left\|x_{n}\right\|_{p}<\infty$,

(ii) $\forall q \in \mathbb{N}_{0} \sup _{n \in \mathbb{N}}\left|\lambda_{n}\right||| x_{n} \|_{q}<\infty$,

then

$$
\forall q \in \mathbb{N}_{0} \forall \theta \in(0,1] \quad \sup _{n \in \mathbb{N}}\left|\lambda_{n}\right|^{\theta}|| x_{n} \|_{q}<\infty .
$$

Moreover, for another sequence $\left(y_{n}\right)_{n \in \mathbb{N}} \subset E$ satisfying conditions (i) and (ii) we have

$$
\forall q \in \mathbb{N}_{0} \forall q^{\prime} \in \mathbb{N}_{0} \forall \theta \in(0,1] \quad \sup _{n \in \mathbb{N}}\left|\lambda_{n}\right|^{\theta}\left\|x_{n}\right\|_{q}\left\|y_{n}\right\|_{q^{\prime}}<\infty .
$$

Proof. Let us fix $q \in \mathbb{N}_{0}$ and $\theta \in(0,1)$. Since $\|\cdot\|_{p}$ is a dominating norm on $E$, thus for some $C>0$ and $r \in \mathbb{N}_{0}$

$$
\left\|x_{n}\right\|_{q} \leq C\left\|x_{n}\right\|_{p}^{1-\theta}\left\|x_{n}\right\|_{r}^{\theta}
$$

for all $n \in \mathbb{N}$. Let $C_{1}:=\sup _{n \in \mathbb{N}}|| x_{n}\left\|_{p}<\infty, C_{2}:=\sup _{n \in \mathbb{N}}\left|\lambda_{n}\right||| x_{n}\right\|_{q}<\infty$. Then by (2)

$$
\left|\lambda_{n}\right|^{\theta}|| x_{n}\left\|_{q} \leq C\right\| x_{n} \|_{p}^{1-\theta}\left(\left|\lambda_{n}\right|\left\|x_{n}\right\|_{r}\right)^{\theta} \leq C C_{1}^{1-\theta} C_{2}^{\theta}=: C_{3},
$$

where $C_{3}$ does not depend on $n$.

To prove the second assertion let us also fix $q^{\prime} \in \mathbb{N}_{0}$ and let $\left(y_{n}\right)_{n \in \mathbb{N}} \subset E$ satisfy conditions (i) and (ii). We have

$$
\left|\lambda_{n}\right|^{\theta}|| x_{n}\left\|_{q}\right\| y_{n} \|_{q^{\prime}}=\left(\left|\lambda_{n}\right|^{\frac{\theta}{2}}|| x_{n} \|_{q}\right)\left(\left|\lambda_{n}\right|^{\frac{\theta}{2}}|| y_{n} \|_{q^{\prime}}\right)
$$

and from the first part of the proof, $\sup _{n \in \mathbb{N}}\left|\lambda_{n}\right|^{\frac{\theta}{2}}\left\|x_{n}\right\|_{q}<\infty$ and $\sup _{n \in \mathbb{N}}\left|\lambda_{n}\right|^{\frac{\theta}{2}}\left\|y_{n}\right\|_{q^{\prime}}<\infty$, so we are done.

Proposition 3.4. Let $\mathcal{N}$ be either a finite set or $\mathcal{N}=\mathbb{N}$. If $\left(P_{n}\right)_{n \in \mathcal{N}}$ is a sequence of pairwise orthogonal finite dimensional projections on $l_{2},\left(\lambda_{n}\right)_{n \in \mathcal{N}} \subset \mathbb{C} \backslash\{0\}$ and $x:=\sum_{n \in \mathcal{N}} \lambda_{n} P_{n} \in$ $L\left(s^{\prime}, s\right)$ (the series converges in norm $\left.\|\cdot\|_{l_{2} \rightarrow l_{2}}\right)$, then $\left(P_{n}\right)_{n \in \mathcal{N}} \subset L\left(s^{\prime}, s\right)$.

Proof. Since, $P_{n}=\frac{1}{\lambda_{n}} x \circ P_{n}$ thus $P_{n}: l_{2} \rightarrow s$, but $P_{n}=P_{n} \circ \frac{1}{\lambda_{n}} x$ so $P_{n}$ extends to $P_{n}: s^{\prime} \rightarrow l_{2}$. Hence, $P_{n}=P_{n} \circ P_{n}: s^{\prime} \rightarrow s$.

Lemma 3.5. Let $\left(\lambda_{n}\right)_{n \in \mathbb{N}}$ be a decreasing (according to the modulus) sequence of nonzero complex numbers and let $\left(P_{n}\right)_{n \in \mathbb{N}}$ be a sequence of nonzero pairwise orthogonal finite dimensional projections on $l_{2}$. Let us also assume that the series $\sum_{n=1}^{\infty} \lambda_{n} P_{n}$ converges in the norm $\|\cdot\|_{l_{2} \rightarrow l_{2}}$ and its limit belongs to $L\left(s^{\prime}, s\right)$. Then $\left(\lambda_{n}\right)_{n \in \mathbb{N}} \in s,\left(P_{n}\right)_{n \in \mathbb{N}} \subset L\left(s^{\prime}, s\right)$ and the series converges absolutely in $L\left(s^{\prime}, s\right)$. Moreover, $\left(\left|\lambda_{n}\right|^{\theta}|| P_{n} \|_{q}\right)_{n \in \mathbb{N}} \in s$ for all $q \in \mathbb{N}_{0}$ and $\theta \in(0,1]$.

Proof. By Prop. 2.4, the sequence of eigenvalues of the operator $x:=\sum_{n=1}^{\infty} \lambda_{n} P_{n}$ belongs to $s$. Clearly, $\lambda_{n}$ is an eigenvalue of $\sum_{n=1}^{\infty} \lambda_{n} P_{n}$ and the number of its occurrences is less or equal to the geometric multiplicity so $\left(\lambda_{n}\right)_{n \in \mathbb{N}}$ is, likewise, in $s$.

By Prop. 3.4, $P_{n} \in L\left(s^{\prime}, s\right)$. We will show that $\left(\left|\lambda_{n}\right|^{\theta}|| P_{n} \|_{q}\right)_{n \in \mathbb{N}} \in s$ for all $q \in \mathbb{N}_{0}$ and $\theta \in(0,1]$, which implies that the series $\sum_{n=1}^{\infty} \lambda_{n} P_{n}$ converges absolutely in $L\left(s^{\prime}, s\right)$. Let us consider operator $T_{x}: L\left(l_{2}\right) \rightarrow L\left(s^{\prime}, s\right)$ which sends $z \in L\left(l_{2}\right)$ to the following composition (in $\left.L\left(s^{\prime}, s\right)\right)$ :

$$
s^{\prime} \stackrel{x}{\rightarrow} s \hookrightarrow l_{2} \stackrel{z}{\rightarrow} l_{2} \hookrightarrow s^{\prime} \stackrel{x}{\rightarrow} s .
$$


By the closed graph theorem for Fréchet spaces (see e.g. [11, Th. 24.31]), $T_{x}$ is continuous and since the sequence of operators $\left(P_{n}\right)_{n \in \mathbb{N}}$ is bounded in $L\left(l_{2}\right)$, thus $\left(\lambda_{n}^{2} P_{n}\right)_{n \in \mathbb{N}}=\left(T_{x} P_{n}\right)_{n \in \mathbb{N}}$ is bounded in $L\left(s^{\prime}, s\right)$, i.e.,

$$
\sup _{n \in \mathbb{N}}\left|\lambda_{n}\right|^{2}|| P_{n} \|_{q}<\infty
$$

for all $q \in \mathbb{N}_{0}$.

Let $\left(e_{n}\right)_{n \in \mathbb{N}}$ be the canonical orthonormal basis in $l_{2}$ and let $E_{n}: s^{\prime} \rightarrow s$,

$$
E_{n} \xi:=\xi_{n} e_{n}
$$

for $\xi=\left(\xi_{n}\right)_{n \in \mathbb{N}} \in s^{\prime}$ and $n \in \mathbb{N}$. Clearly, $E_{n}$ are projections in $L\left(s^{\prime}, s\right)$. Moreover,

$$
\left\|E_{n}\right\|_{q}=\sup _{|\xi|_{q}^{\prime} \leq 1}\left|E_{n} \xi\right|_{q}=\sup _{|\xi|_{q}^{\prime} \leq 1}\left|\xi_{n} e_{n}\right|_{q}=\sup _{|\xi|_{q}^{\prime} \leq 1}\left|\xi_{n}\right| \cdot\left|e_{n}\right|_{q}=n^{q} \cdot n^{q}=n^{2 q}
$$

Since $\left(\lambda_{n}\right)_{n \in \mathbb{N}} \in s$, thus

$$
\sup _{n \in \mathbb{N}}\left|\lambda_{n}\right|^{2}|| E_{n} \|_{q}<\infty
$$

for $q \in \mathbb{N}_{0}$

By Proposition 3.2. $\|\cdot\| \|_{l_{2} \rightarrow l_{2}}$ is a dominating norm on $L\left(s^{\prime}, s\right)$, and, of course, $\left\|P_{n}\right\|_{l_{2} \rightarrow l_{2}}=$ $\left\|E_{n}\right\|_{l_{2} \rightarrow l_{2}}=1$ for $n \in \mathbb{N}$. Thus, from inequalities (3), (5)), equality (4) and by Lemma 3.3 (applied to sequences $\left(\lambda_{n}^{2}\right)_{n \in \mathbb{N}},\left(P_{n}\right)_{n \in \mathbb{N}}$ and $\left.\left(E_{n}\right)_{n \in \mathbb{N}}\right)$ we get

$$
\sup _{n \in \mathbb{N}}\left|\lambda_{n}\right|^{2 \theta}|| P_{n}\left\|_{q} n^{2 q^{\prime}}=\sup _{n \in \mathbb{N}}\left|\lambda_{n}\right|^{2 \theta}|| P_{n}\right\|_{q}|| E_{n} \|_{q^{\prime}}<\infty
$$

for all $\theta \in(0,1]$ and $q, q^{\prime} \in \mathbb{N}_{0}$. Hence, $\left(\left|\lambda_{n}\right|^{\theta}\left\|P_{n}\right\|_{q}\right) \in s$ for all $q \in \mathbb{N}_{0}$ and $\theta \in(0,1]$.

Now, it is not hard to prove the main theorem of this section.

Proof of Theorem 3.1. Let $x$ be a normal infinite dimensional operator in $L\left(s^{\prime}, s\right)$. Operator $x$ (as an operator on $l_{2}$ ) is compact (see [6, Prop. 3.1]), thus by the spectral theorem for normal compact operators (see e.g. [4, Th. 7.6]), $x=\sum_{n=1}^{\infty} \lambda_{n} P_{n}$, where $\left(\lambda_{n}\right)_{n \in \mathbb{N}}$ is a decreasing zero sequence of nonzero pairwise different elements, $\left(P_{n}\right)_{n \in \mathbb{N}}$ is a sequence of nonzero pairwise orthogonal finite dimensional projections and the series converges in norm $\|\cdot\|_{l_{2} \rightarrow l_{2}}$. Now, the conclusion follows by Lemma 3.5 .

As a corollary, we get a characterization of normal operators in $L\left(s^{\prime}, s\right)$ among compact operators on $l_{2}$.

Corollary 3.6. Let $x$ be a compact infinite dimensional normal operator on $l_{2}$ with the spectral representation $x=\sum_{n=1}^{\infty} \lambda_{n} P_{n}$ (the series converges in norm $\|\cdot\|_{l_{2} \rightarrow l_{2}}$ ). Then the following assertions are equivalent:

(i) $x \in L\left(s^{\prime}, s\right)$ (as an operator on $\left.l_{2}\right)$;

(ii) $P_{n} \in L\left(s^{\prime}, s\right)$ for $n \in \mathbb{N}$ and $\left(\left|\lambda_{n}\right|^{\theta}|| P_{n} \|_{q}\right)_{n \in \mathbb{N}} \in s$ for all $q \in \mathbb{N}_{0}$ and every $\theta \in(0,1]$;

(iii) $P_{n} \in L\left(s^{\prime}, s\right)$ for $n \in \mathbb{N},\left(\lambda_{n}\right)_{n \in \mathbb{N}} \in s$ and $\sup _{n \in \mathbb{N}}\left|\lambda_{n}\right|\left\|P_{n}\right\|_{q}<\infty$ for all $q \in \mathbb{N}_{0}$;

(iv) $P_{n} \in L\left(s^{\prime}, s\right)$ for $n \in \mathbb{N}$ and $\sum_{n=1}^{\infty}\left|\lambda_{n}\right|\left\|P_{n}\right\|_{q}<\infty$ for all $q \in \mathbb{N}_{0}$.

Moreover, if $x=\sum_{n=1}^{N} \lambda_{n} P_{n}$ is a finite dimensional operator on $l_{2}$, then $x \in L\left(s^{\prime}, s\right)$ if and only if $P_{n} \in L\left(s^{\prime}, s\right)$ for $n=1, \ldots, N$. 
Proof. The implication (i) $\Rightarrow$ (ii) follows directly by Theorem 3.1 . The implications (ii) $\Rightarrow$ (iii), (iv) $\Rightarrow$ (i) are obvious.

(iii) $\Rightarrow$ (iv) By Lemma 3.3, $\sup _{n \in \mathbb{N}}\left|\lambda_{n}\right|^{\frac{1}{2}}|| P_{n} \|_{q}<\infty$, and, moreover, $\sum_{n=1}^{\infty}\left|\lambda_{n}\right|^{\frac{1}{2}}<\infty(s \subset$ $\left.\bigcap_{p>0} l_{p}\right)$. Thus

$$
\sum_{n=1}^{\infty}\left|\lambda_{n}\right||| P_{n} \|_{q} \leq\left.\sup _{n \in \mathbb{N}}\left|\lambda_{n}\right|^{\frac{1}{2}}|| P_{n}\right|_{q} \cdot \sum_{n=1}^{\infty}\left|\lambda_{n}\right|^{\frac{1}{2}}<\infty,
$$

and the remaining implication (iii) $\Rightarrow$ (iv) holds.

The finite case is an immediate consequence of Proposition 3.4 .

\section{Closed commutative ${ }^{*}$-subalgebras of $L\left(s^{\prime}, s\right)$}

The aim of this section is to describe all closed commutative ${ }^{*}$-subalgebras of $L\left(s^{\prime}, s\right)$ (see Theorem 4.8) and identifying maximal among them (see Theorem 4.10).

We will need the following lemma.

Lemma 4.1. Let $A$ be a subalgebra of the algebra $\widetilde{A}$ over $\mathbb{C}$. Let $N \in \mathbb{N}, a_{1}, \ldots, a_{N} \in \widetilde{A}$, $\lambda_{1}, \ldots, \lambda_{N} \in \mathbb{C}, a_{j} \neq 0, a_{j}^{2}=a_{j}, a_{j} a_{k}=0$ for $j \neq k, \lambda_{j} \neq 0$ and $\lambda_{j} \neq \lambda_{k}$ for $j \neq k$. Then $a_{1}, \ldots, a_{N} \in A$ whenever $\lambda_{1} a_{1}+\ldots+\lambda_{N} a_{N} \in A$.

Proof. We use induction with respect to $N$. The case $N=1$ is trivial.

Let us assume that the conlusion holds for all $M<N$. Let $a:=\lambda_{1} a_{1}+\ldots+\lambda_{N} a_{N} \in A$. We have

$$
\lambda_{1}^{2} a_{1}+\ldots+\lambda_{N}^{2} a_{N}=a^{2} \in A
$$

and, on the other hand,

$$
\lambda_{N} \lambda_{1} a_{1}+\ldots+\lambda_{N}^{2} a_{N}=\lambda_{N} a \in A
$$

so

$$
\left(\lambda_{1}^{2}-\lambda_{N} \lambda_{1}\right) a_{1}+\ldots+\left(\lambda_{N-1}^{2}-\lambda_{N} \lambda_{N-1}\right) a_{N-1}=a^{2}-\lambda_{N} a \in A .
$$

Since $\lambda_{j} \neq 0$ and $\lambda_{j} \neq \lambda_{N}$ for $j \in\{1, \ldots, N-1\}$, thus $\lambda_{j}^{2}-\lambda_{N} \lambda_{j}=\lambda_{j}\left(\lambda_{j}-\lambda_{N}\right) \neq 0$ for $j \in\{1, \ldots, N-1\}$. If $\lambda_{j}^{2}-\lambda_{N} \lambda_{j}$ are pairwise different then, from the inductive assumption, $a_{1}, \ldots, a_{N-1} \in A$ so $a_{N} \in A$ as well.

Let us assume that it is not the case. We define the equivalence relation $\mathcal{R}$ on the set $\{1, \ldots, N-1\}$ in the following way

$$
j \mathcal{R} k \Leftrightarrow \lambda_{j}\left(\lambda_{j}-\lambda_{N}\right)=\lambda_{k}\left(\lambda_{k}-\lambda_{N}\right)
$$

Let $I_{1}, \ldots, I_{N_{1}}$ denote the equivalence classes which contain not less than two elements and let $I_{0}:=\left\{i_{1}, \ldots, i_{N_{0}}\right\}$ be the set of indices from the remaining equivalence classes. From our assumption, $I_{1} \neq \emptyset$. For $j \in\left\{1, \ldots, N_{1}\right\}, k \in I_{j}$ let

$$
\lambda_{j}^{\prime}:=\lambda_{k}\left(\lambda_{k}-\lambda_{N}\right)
$$

and let

$$
a_{j}^{\prime}:=\sum_{n \in I_{j}} a_{n}
$$

We also define

$$
\lambda_{N_{1}+1}^{\prime}:=\lambda_{i_{1}}\left(\lambda_{i_{1}}-\lambda_{N}\right), \lambda_{N_{1}+2}^{\prime}:=\lambda_{i_{2}}\left(\lambda_{i_{2}}-\lambda_{N}\right), \ldots, \lambda_{N_{1}+N_{0}}^{\prime}:=\lambda_{i_{N_{0}}}\left(\lambda_{i_{N_{0}}}-\lambda_{N}\right)
$$


and

$$
a_{N_{1}+1}^{\prime}:=a_{i_{1}}, a_{N_{1}+2}^{\prime}:=a_{i_{2}}, \ldots, a_{N_{1}+N_{0}}^{\prime}:=a_{i_{N_{0}}} .
$$

Clearly, $1 \leq N^{\prime}:=N_{1}+N_{0}<N, a_{j}^{\prime} \neq 0, a_{j}^{\prime 2}=a_{j}^{\prime}, a_{j}^{\prime} a_{k}^{\prime}=0, \lambda_{j}^{\prime} \neq 0, \lambda_{j}^{\prime} \neq \lambda_{k}^{\prime}$ for $j, k \in$ $\left\{1, \ldots, N^{\prime}\right\}, j \neq k$ and

$$
\lambda_{1}^{\prime} a_{1}^{\prime}+\ldots+\lambda_{N^{\prime}}^{\prime} a_{N^{\prime}}^{\prime}=a^{2}-\lambda_{N} a \in A .
$$

From the inductive assumption, $a_{1}^{\prime} \in A$, hence

$$
\sum_{n \in I_{1}} \lambda_{n} a_{n}=\sum_{n \in I_{1}} a_{n} \cdot \sum_{n=1}^{N} \lambda_{n} a_{n}=a_{1}^{\prime} a \in A .
$$

Again, from the inductive assumption, $a_{n} \in A$ for $n \in I_{1}$, and, therefore, $\sum_{n \in\{1, \ldots, N\} \backslash I_{1}} \lambda_{n} a_{n} \in$ $A$. Once again, from the inductive assumption, $a_{n} \in A$ for $n \in\{1, \ldots, N\} \backslash I_{1}$. Thus $a_{1}, \ldots, a_{N} \in$ $A$ which completes the proof.

Proposition 4.2. Let $A$ be a closed ${ }^{*}$-subalgebra of $L\left(s^{\prime}, s\right)$ (not necessary commutative) and let $x$ be an infinite dimensional normal operator in $L\left(s^{\prime}, s\right)$ with the spectral representation $x=\sum_{n=1}^{\infty} \lambda_{n} P_{n}$. Then $x \in A$ if and only if $P_{n} \in A$ for all $n \in \mathbb{N}$.

Proof. By Theorem 3.1, if $P_{n} \in A$ for all $n \in \mathbb{N}$ then $x \in A$. To prove the converse let us assume that $x \in A$. Then $x^{*}=\sum_{n=1}^{\infty} \overline{\lambda_{n}} P_{n} \in A$ so $x x^{*}=\sum_{n=1}^{\infty}\left|\lambda_{n}\right|^{2} P_{n} \in A$. Let $N_{0}:=0$, $N_{1}:=\sup \left\{n \in \mathbb{N}:\left|\lambda_{n}\right|=\left|\lambda_{1}\right|\right\}$ and for $j=2,3, \ldots$, let $N_{j}:=\sup \left\{n \in \mathbb{N}:\left|\lambda_{n}\right|=\left|\lambda_{N_{j-1}+1}\right|\right\}$. Since $\left(\left|\lambda_{n}\right|\right)_{n \in \mathbb{N}}$ is a zero sequence thus $N_{j}<\infty$. We have

$$
y_{k}:=\left(\frac{x x^{*}}{\left|\lambda_{1}\right|^{2}}\right)^{k}=\sum_{n=1}^{\infty}\left(\frac{\left|\lambda_{n}\right|}{\left|\lambda_{1}\right|}\right)^{2 k} P_{n} \in A
$$

for all $k \in \mathbb{N}$. For $q$ and $k$ arbitrary, we get

$$
\begin{aligned}
\left\|y_{k}-\left(P_{1}+\ldots+P_{N_{1}}\right)\right\|_{q} & =\left\|\sum_{n=1}^{\infty}\left(\frac{\left|\lambda_{n}\right|}{\left|\lambda_{1}\right|}\right)^{2 k} P_{n}-\left(P_{1}+\ldots+P_{N_{1}}\right)\right\|_{q}=\left\|\sum_{n=N_{1}+1}^{\infty}\left(\frac{\left|\lambda_{n}\right|}{\left|\lambda_{1}\right|}\right)^{2 k} P_{n}\right\|_{q} \\
& \leq \sum_{n=N_{1}+1}^{\infty}\left(\frac{\left|\lambda_{n}\right|}{\left|\lambda_{1}\right|}\right)^{2 k}\left\|P_{n}\right\|_{q} \leq \frac{1}{\left|\lambda_{1}\right|}\left(\frac{\left|\lambda_{N_{1}+1}\right|}{\left|\lambda_{1}\right|}\right)^{2 k-1} \sum_{n=N_{1}+1}^{\infty}\left|\lambda_{n}\right|\left\|P_{n}\right\|_{q} .
\end{aligned}
$$

By Theorem 3.1, $\sum_{n=N_{1}+1}^{\infty}\left|\lambda_{n}\right||| P_{n} \|_{q}<\infty$, and, moreover, $\frac{\left|\lambda_{N_{1}+1}\right|}{\left|\lambda_{1}\right|}<1$, thus

$$
\left\|y_{k}-\left(P_{1}+\ldots+P_{N_{1}}\right)\right\|_{q} \rightarrow 0
$$

as $k \rightarrow \infty$. Therefore, since $A$ is closed, we conlude that $P_{1}+\ldots+P_{N_{1}} \in A$. Consequently,

$$
\sum_{n=N_{1}+1}^{\infty}\left|\lambda_{n}\right|^{2} P_{n}=x x^{*}-\left|\lambda_{1}\right|^{2}\left(P_{1}+\ldots+P_{N_{1}}\right) \in A
$$

hence, proceeding by induction, we get that $P_{N_{j}+1}+\ldots P_{N_{j+1}} \in A$ for $j \in \mathbb{N}_{0}$ so

$$
\sum_{n=N_{j}+1}^{N_{j+1}} \lambda_{n} P_{n}=\left(P_{N_{j}+1}+\ldots P_{N_{j+1}}\right) x \in A .
$$

Finally, by Lemma 4.1, $P_{n} \in A$ for $n \in \mathbb{N}$ 
Proposition 4.3. For every othonormal system $\left(e_{n}\right)_{n \in \mathbb{N}}$ in $l_{2}$ and sequence $\left(\lambda_{n}\right)_{n \in \mathbb{N}} \in c_{0}$, the series $\sum_{n=1}^{\infty} \lambda_{n}\left\langle\cdot, e_{n}\right\rangle e_{n}$ converges in norm $\|\cdot\|_{l_{2} \rightarrow l_{2}}$.

Proof. This is a simple consequence of the Pythagorean theorem and the Bessel's inequality.

Lemma 4.4. Let $A$ be a commutative subalgebra of $L\left(s^{\prime}, s\right)$. Let $\mathcal{P}$ denote the set of nonzero projections belonging to $A$ and let $\mathcal{M}$ be the set of minimal elements in $\mathcal{P}$ with respect to the following order relation

$$
\forall P, Q \in \mathcal{P} \quad P \preceq Q \Leftrightarrow P Q=Q P=P .
$$

Then

(i) $\mathcal{M}$ is at most countable family of pairwise orthogonal projections belonging to $L\left(s^{\prime}, s\right)$ such that

$$
\forall P \in \mathcal{P} \exists P_{1}^{\prime}, \ldots, P_{m}^{\prime} \in \mathcal{M} \quad P=P_{1}^{\prime}+\cdots+P_{m}^{\prime} .
$$

(ii) If $A$ is also a closed ${ }^{*}$-subalgebra of $L\left(s^{\prime}, s\right)$, then $\mathcal{M}$ is a Schauder basis in $A$.

Proof. (i) By the definition

$$
\mathcal{M}=\{P \in \mathcal{P}: \forall Q \in \mathcal{P} \quad(Q \preceq P \Rightarrow Q=P)\} .
$$

Firstly, we will show that

$$
\forall P \in \mathcal{P} \exists P_{1}^{\prime}, \ldots, P_{m}^{\prime} \in \mathcal{M} \quad P=P_{1}^{\prime}+\cdots+P_{m}^{\prime} .
$$

Let us take $P \in \mathcal{P}$. If $P \in \mathcal{M}$, then we are done. Otherwise, there is $Q \in \mathcal{P}$ such that $Q \preceq P, Q \neq P$. Of course, $P-Q \in \mathcal{P}$. If $Q, P-Q \in \mathcal{M}$, then $P=Q+(P-Q)$ is desired decomposition. Otherwise, we decompose $Q$ or $P-Q$ into smaller projections as it was done above for projection $P$. Since $P$ is finite dimensional thus after finitely many steps we finish our procedure.

Next, we shall prove that projections in $\mathcal{M}$ are pairwise orthogonal. Let $P, Q \in \mathcal{M}, P \neq Q$ and let us suppose, to derive a contradiction, that $P Q \neq 0$. Since $A$ is commutative, thus

$$
(P Q)^{2}=P^{2} Q^{2}=P Q
$$

so $P Q \in \mathcal{P}$. Moreover,

$$
P(P Q)=P^{2} Q=P Q
$$

so $P Q \preceq P$. Now, $P Q \neq P$ implies that $P \notin \mathcal{M}$ and if $P Q=P$ then $Q \notin \mathcal{M}$, which is a contradiction.

Finally, since projections in $\mathcal{M}$ are pairwise orthogonal (as projections on $l_{2}$ ) thus $\mathcal{M}$ is at most countable.

(ii) Let $x \in A$. If $x$ is finite dimensional and $\sum_{n=1}^{N} \mu_{n} Q_{n}$ is its spectral decomposistion, then from (i) and by Lemma 4.1, $x$ is a linear combination of projections in $\mathcal{M}$.

Let us assume that $x$ is infinite dimensional and let $x=\sum_{n=1}^{\infty} \mu_{n} Q_{n}$ (the spectral representation of $x$ ). Since $A$ is a closed commutative ${ }^{*}$-subalgebra of $L\left(s^{\prime}, s\right)$ thus, by Proposition 4.2, $Q_{n} \in A$ for $n \in \mathbb{N}$. Next, from (i), we have that

$$
\forall n \in \mathbb{N} \exists Q_{1}^{(n)}, \ldots, Q_{l_{n}}^{(n)} \in \mathcal{M} \quad Q_{n}=\sum_{j=1}^{l_{n}} Q_{j}^{(n)} .
$$


Hence

$$
x=\sum_{n=1}^{\infty} \sum_{j=1}^{l_{n}} \mu_{n} Q_{j}^{(n)} .
$$

For $l_{0}=0, j=l_{0}+l_{1}+\ldots+l_{n-1}+k, 1 \leq k \leq l_{n}$ let $P_{j}:=Q_{k}^{(n)}$ and let $\lambda_{j}:=\mu_{n}$. Let us consider the series $\sum_{n=1}^{\infty} \lambda_{n} P_{n}$. Clearly, if the series converges in $L\left(s^{\prime}, s\right)$ then its limit is $x$. To prove this we shall firstly show that the series $\sum_{n=1}^{\infty} \lambda_{n} P_{n}$ converges in the norm $\|\cdot\|_{l_{2} \rightarrow l_{2}}$.

Since $P_{n}$ is a (orthogonal) projection of finite dimension $d_{n}$ thus $P_{n}=\sum_{j=1}^{d_{n}}\left\langle\cdot, e_{j}^{(n)}\right\rangle e_{j}^{(n)}$ for every orthonormal basis $\left(e_{j}^{(n)}\right)_{j=1}^{d_{n}}$ of the image of $P_{n}$. For $d_{0}=0, j=d_{0}+d_{1}+\ldots+d_{n-1}+k$, $1 \leq k \leq d_{n}$ let $e_{j}:=e_{k}^{(n)}$ and let $\lambda_{j}^{\prime}:=\lambda_{n}$. By Proposition 4.3, the series $\sum_{j=1}^{\infty} \lambda_{j}^{\prime}\left\langle\cdot, e_{j}\right\rangle e_{j}$ converges in the norm $\|\cdot\|_{l_{2} \rightarrow l_{2}}$. Hence $\sum_{n=1}^{\infty} \lambda_{n} P_{n}$ converges in the norm $\|\cdot\|_{l_{2} \rightarrow l_{2}}$ because $\left(\sum_{n=1}^{N} \lambda_{n} P_{n}\right)_{N \in \mathbb{N}}$ is a subsequence of the sequence of partial sums of the series $\sum_{j=1}^{\infty} \lambda_{j}^{\prime}\left\langle\cdot, e_{j}\right\rangle e_{j}$.

Now, by Lemma 3.5, $x=\sum_{n=1}^{\infty} \lambda_{n} P_{n}$ and the series converges absolutely in $L\left(s^{\prime}, s\right)$. This show that every operator in $A$ is represented by the absolutely convergent series $\sum_{n=1}^{\infty} \lambda_{n}^{\prime \prime} P_{n}^{\prime \prime}$ with $P_{n}^{\prime \prime} \in \mathcal{M}$. To prove the uniquness of this representation let us assume that $\sum_{n=1}^{\infty} \lambda_{n}^{\prime \prime} P_{n}^{\prime \prime}=0$. Then

$$
\lambda_{m}^{\prime \prime} P_{m}^{\prime \prime}=P_{m}^{\prime \prime} \sum_{n=1}^{\infty} \lambda_{n}^{\prime \prime} P_{n}^{\prime \prime}=0
$$

so $\lambda_{m}^{\prime \prime}=0$ for $m \in \mathbb{N}$. This show that the sequence of coefficients is unique, hence $\mathcal{M}$ is a Schauder basis in $A$.

For a closed commutative *-subalgebra $A$ of the algebra $L\left(s^{\prime}, s\right)$ the Schauder basis $\mathcal{M}$ from the preceeding lemma will be called the canonical Schauder basis (of $A$ ).

For a subset $Z$ of $L\left(s^{\prime}, s\right)$ we will denote by $\operatorname{alg}(Z)$ the closed ${ }^{*}$-subalgebra of $L\left(s^{\prime}, s\right)$ generated by $Z$. If $A$ is a closed *-subalgebra of $L\left(s^{\prime}, s\right)$, then $\widehat{A}$ denotes the set of nonzero ${ }^{*}$-multiplicative functionals on $A$.

Corollary 4.5. The set $\widehat{A}$ of nonzero ${ }^{*}$-multiplicative functionals on a closed commutative *subalgebra $A$ of $L\left(s^{\prime}, s\right)$ is exactly the set of coefficient functionals with respect to the canonical Schauder basis of $A$.

Proof. Clearly, every coefficient functional is *-multiplicative. Conversly, if $\varphi$ is a nonzero *multiplicative functional on $A$ and $\left\{P_{n}\right\}_{n \in \mathbb{N}}$ is the canonical Schauder basis then $\varphi\left(P_{n}\right)=$ $\varphi\left(P_{n}^{2}\right)=\left(\varphi\left(P_{n}\right)\right)^{2}$, thus $\varphi\left(P_{n}\right)=0$ or $\varphi\left(P_{n}\right)=1$. Let us suppose that $\varphi\left(P_{n}\right)=\varphi\left(P_{m}\right)=1$ for $n \neq m$. Then

$$
\begin{aligned}
2 & =\varphi\left(P_{n}\right)+\varphi\left(P_{m}\right)=\varphi\left(P_{n}+P_{m}\right)=\varphi\left(\left(P_{n}+P_{m}\right)^{2}\right)=\left(\varphi\left(P_{n}+P_{m}\right)\right)^{2} \\
& =\left(\varphi\left(P_{n}\right)+\varphi\left(P_{m}\right)\right)^{2}=4,
\end{aligned}
$$

a contradiction. Hence, there is at most one $n \in \mathbb{N}$ such that $\varphi\left(P_{n}\right)=1$. If $\varphi\left(P_{n}\right)=0$ for all $n \in \mathbb{N}$ then, since $\left\{P_{n}\right\}_{n \in \mathbb{N}}$ is a basis, $\varphi=0$, a contradiction. Thus, there is exactly one $n \in \mathbb{N}$ such that $\varphi\left(P_{n}\right)=1$, and $\varphi\left(P_{m}\right)=0$ for $m \neq n$, i.e., $\varphi$, is a coefficient functional.

Proposition 4.6. If $\left\{P_{n}\right\}_{n \in \mathcal{N}}$ is a family of pairwise orthogonal projections belonging to $L\left(s^{\prime}, s\right)$, then

$$
\operatorname{alg}\left(\left\{P_{n}\right\}_{n \in \mathcal{N}}\right)=\varlimsup \operatorname{lin}\left(\left\{P_{n}\right\}_{n \in \mathcal{N}}\right)
$$

and it is a commutative ${ }^{*}$-algebra. 
Proof. Clearly, $\overline{\operatorname{lin}}\left(\left\{P_{n}\right\}_{n \in \mathcal{N}}\right) \subseteq \operatorname{alg}\left(\left\{P_{n}\right\}_{n \in \mathcal{N}}\right)$ and $\operatorname{lin}\left(\left\{P_{n}\right\}_{n \in \mathcal{N}}\right)$ is a commutative ${ }^{*}$-algebra. By the continuity of the algebra multiplication and the hilbertian involution, $\overline{\operatorname{lin}}\left(\left\{P_{n}\right\}_{n \in \mathcal{N}}\right)$ is a commutative *-algebra as well. Hence, $\overline{\operatorname{lin}}\left(\left\{P_{n}\right\}_{n \in \mathcal{N}}\right)=\operatorname{alg}\left(\left\{P_{n}\right\}_{n \in \mathcal{N}}\right)$.

Proposition 4.7. Every sequence $\left\{P_{n}\right\}_{n \in \mathcal{N}} \subset L\left(s^{\prime}, s\right)$ of nonzero pairwise orthogonal projections is a basic sequence in $L\left(s^{\prime}, s\right)$, i.e., it is a (canonical) Schauder basis of the Fréchet space $\left({ }^{*}\right.$-algebra) $\overline{\operatorname{lin}}\left(\left\{P_{n}\right\}_{n \in \mathcal{N}}\right)$.

Proof. Let $\mathcal{M}$ be the canonical Schauder basis of $A:=\operatorname{alg}\left(\left\{P_{n}\right\}_{n \in \mathcal{N}}\right)$ which consists of all projections which are minimal with respect to the order relation described in Lemma 4.4. If we show that $\left\{P_{n}\right\}_{n \in \mathcal{N}}=\mathcal{M}$, then, by Proposition 4.6, we get the conclusion.

Let $n \in \mathcal{N}$ be fixed and let us assume that $Q \preceq P_{n}$ for some nonzero projection $Q \in A$, i.e., $Q P_{n}=Q$. Since $A=\overline{\operatorname{lin}}\left(\left\{P_{n}\right\}_{n \in \mathcal{N}}\right)$ thus

$$
Q=\lim _{j \rightarrow \infty} \sum_{k=1}^{M_{j}} \lambda_{k}^{(j)} P_{k}
$$

for some $M_{j} \in \mathbb{N}$ and $\lambda_{k}^{(j)} \in \mathbb{C}$. From the continuity of the algebra and scalar multiplication, we get

$$
\begin{aligned}
Q=Q P_{n} & =\left(\lim _{j \rightarrow \infty} \sum_{k=1}^{M_{j}} \lambda_{k}^{(j)} P_{k}\right) P_{n}=\lim _{j \rightarrow \infty}\left(\sum_{k=1}^{M_{j}} \lambda_{k}^{(j)} P_{k} P_{n}\right)=\lim _{j \rightarrow \infty}\left(\lambda_{n}^{(j)} P_{n}\right) \\
& =\left(\lim _{j \rightarrow \infty} \lambda_{n}^{(j)}\right) P_{n}=\lambda_{n} P_{n}
\end{aligned}
$$

where $\lambda_{n}:=\lim _{j \rightarrow \infty} \lambda_{n}^{(j)} \in \mathbb{C}$. Since $Q$ is a nonzero projection, thus $\lambda_{n}=1$ and $Q=P_{n}$. Hence $\left\{P_{n}\right\}_{n \in \mathcal{N}} \subseteq \mathcal{M}$.

Now, let us suppose that there is a projection $Q$ in $\mathcal{M} \backslash\left\{P_{n}\right\}_{n \in \mathcal{N}}$. We have already proved that $\left\{P_{n}\right\}_{n \in \mathcal{N}} \subseteq \mathcal{M}$, hence by Lemma 4.4 (i), $Q x=0$ for all $x \in \operatorname{lin}\left(\left\{P_{n}\right\}_{n \in \mathcal{N}}\right)$. By continuity of the multiplication, $Q x=0$ for every $x \in \overline{\operatorname{lin}}\left(\left\{P_{n}\right\}_{n \in \mathcal{N}}\right)=A$. In particular, $Q=Q^{2}=0$, a contradiction. Hence, $\left\{P_{n}\right\}_{n \in \mathcal{N}}=\mathcal{M}$.

Closed commutative ${ }^{*}$-subalgebras of $L\left(s^{\prime}, s\right)$ are quite simple, all of them are generated by a single operator and also by its spectral projections. From nuclearity we get also the following sequence space representations.

Theorem 4.8. Let $A$ be a closed commutative infinite dimensional ${ }^{*}$-subalgebra of $L\left(s^{\prime}, s\right)$. Then $A$ has a (canonical) Schauder basis $\left\{P_{n}\right\}_{n \in \mathbb{N}}$ consisting of pairwise othogonal finite dimensional minimal projections (see Lemma 4.4) such that

$$
A=\operatorname{alg}\left(\left\{P_{n}\right\}_{n \in \mathbb{N}}\right) \cong \lambda^{1}\left(\left\|P_{n}\right\|_{q}\right)=\lambda^{\infty}\left(\left\|P_{n}\right\|_{q}\right)
$$

as Fréchet ${ }^{*}$-algebras. Moreover, there is an operator $x \in A$ with the spectral representation $x=\sum_{n=1}^{\infty} \lambda_{n} P_{n}$ such that $A=\operatorname{alg}(x)$.

Proof. By Lemma 4.4, $A$ has a Schauder basis with the desired properties. By Proposition 4.6, $A=\overline{\operatorname{lin}}\left(\left\{P_{n}\right\}_{n \in \mathbb{N}}\right)=\operatorname{alg}\left(\left\{P_{n}\right\}_{n \in \mathbb{N}}\right)$ and since $A$ is a nuclear Fréchet space with the Schauder basis $\left\{P_{n}\right\}_{n \in \mathbb{N}}$, thus (see e.g. [11, Cor. 28.13, Prop. 28.16])

$$
A \cong \lambda^{1}\left(\left\|P_{n}\right\|_{q}\right)=\lambda^{\infty}\left(\left\|P_{n}\right\|_{q}\right)
$$


as Fréchet spaces. Since on linear span of $\left\{P_{n}\right\}_{n \in \mathbb{N}}$ the multiplication (involution) corresponds to the pointwise multiplication (conjugation) in $\lambda^{1}\left(\left\|P_{n}\right\|_{q}\right)$ thus the isomorphism is also *algebra isomorphism where the Köthe space is equipped with the pointwise multiplication.

Now, we shall show that there is a decreasing sequence $\left(\lambda_{n}\right)_{n \in \mathbb{N}}$ of positive numbers such that the series $\sum_{n=1}^{\infty} \lambda_{n} P_{n}$ is absolutely convergent in $L\left(s^{\prime}, s\right)$. To do so, let us choose a sequence $\left(C_{q}\right)_{q \in \mathbb{N}}$ such that $C_{q} \geq \max _{1 \leq n \leq q}\left\|P_{n}\right\|_{q}$. Clearly, $\frac{C_{q}}{\left\|P_{n}\right\|_{q}} \geq 1$ for $q \geq n$ so

$$
\inf _{q \in \mathbb{N}} \frac{C_{q}}{\left\|P_{n}\right\|_{q}} \geq \min \left\{\frac{C_{1}}{\left\|P_{n}\right\|_{1}}, \ldots, \frac{C_{n-1}}{\left\|P_{n}\right\|_{n-1}}, 1\right\}>0
$$

for $n \in \mathbb{N}$. Let $\lambda_{1}:=1$ and let

$$
\lambda_{n}:=\min \left\{\frac{1}{n^{2}} \inf _{q \in \mathbb{N}} \frac{C_{q}}{\left\|P_{n}\right\|_{q}}, \frac{\lambda_{n-1}}{2}\right\} .
$$

Then $\lambda_{n}>0$, the sequence $\left(\lambda_{n}\right)_{n \in \mathbb{N}}$ is strictly decreasing and

$$
\sum_{n=1}^{\infty} \lambda_{n}\left\|P_{n}\right\|_{q} \leq \sum_{n=1}^{\infty} \frac{1}{n^{2}} \inf _{r \in \mathbb{N}} \frac{C_{r}}{\left\|P_{n}\right\|_{r}}\left\|P_{n}\right\|_{q} \leq C_{q} \sum_{n=1}^{\infty} \frac{1}{n^{2}}<\infty .
$$

Consequently, $x:=\sum_{n=1}^{\infty} \lambda_{n} P_{n} \in L\left(s^{\prime}, s\right)$ and this series is the spectral representation of $x$. Moreover, since $P_{n} \in A$ for $n \in \mathbb{N}$ and $A$ is closed, thus $x \in A$. Finally, the equality $\operatorname{alg}(x)=\operatorname{alg}\left(\left\{P_{n}\right\}_{n \in \mathbb{N}}\right)$ is a consequence of Proposition 4.2

A commutative closed *-subalgebra $A$ of the algebra $L\left(s^{\prime}, s\right)$ is said to be maximal commutative if it is not contain in any larger closed commutative ${ }^{*}$-subalgebra of $L\left(s^{\prime}, s\right)$. We say that the sequence $\left\{P_{n}\right\}_{n \in \mathbb{N}}$ of nonzero pairwise orthogonal projections in $L\left(s^{\prime}, s\right)$ is complete if there is no nonzero projection $P$ in $L\left(s^{\prime}, s\right)$ such that $P_{n} P=0$ for every $n \in \mathbb{N}$. For a subset $Z$ of the algebra $L\left(s^{\prime}, s\right)$, the set $Z^{\prime}:=\left\{x \in L\left(s^{\prime}, s\right): x y=y x\right.$ for all $\left.y \in Z\right\}$ is called the commutant of $Z$.

Proposition 4.9. For every subset $Z$ of $L\left(s^{\prime}, s\right)$, the comutant $Z^{\prime}$ is a closed ${ }^{*}$-subalgebra of $L\left(s^{\prime}, s\right)$.

Proof. Clearly, if $x, y$ commute with every $z \in Z$ then $\lambda x, x+y, x y$ and $x^{*}$ commute as well. Hence, from the continuity of the algebra operations and the involution, $Z^{\prime}$ is a closed ${ }^{*}$-subalgebra of $L\left(s^{\prime}, s\right)$.

Theorem 4.10. For a closed commutative ${ }^{*}$-subalgebra $A$ of $L\left(s^{\prime}, s\right)$ the following assertions are equivalent:

(i) A is maximal commutative;

(ii) The canonical Schauder basis $\left\{P_{n}\right\}_{n \in \mathbb{N}}$ of $A$ is a complete sequence of pairwise orthogonal one-dimensional projections belonging to $L\left(s^{\prime}, s\right)$;

(iii) $A=A^{\prime}$.

Proof. (i) $\Rightarrow$ (ii). Let us suppose that for some $m \in \mathbb{N}$ the projection $P_{m}$ is not one dimensional. Then there are two nonzero pairwise orthogonal projections $Q_{1}, Q_{2} \in L\left(s^{\prime}, s\right)$ such that $P_{m}=$ $Q_{1}+Q_{2}$. By Proposition 4.6, $\overline{\operatorname{lin}}\left(\left\{P_{n}: n \neq m\right\} \cup\left\{Q_{1}, Q_{2}\right\}\right)$ is a closed commutative ${ }^{*}$-subalgebra of $L\left(s^{\prime}, s\right)$, and, clearly,

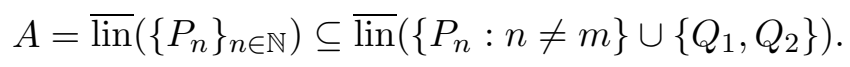


By Proposition 4.7, $\left\{P_{n}\right\}_{n \in \mathbb{N}}$ is the canonical Schauder basis of $A$ and $\left\{P_{n}: n \neq m\right\} \cup\left\{Q_{1}, Q_{2}\right\}$ is the canonical Schauder basis of $\varlimsup\left(\left\{P_{n}: n \neq m\right\} \cup\left\{Q_{1}, Q_{2}\right\}\right)$ so

$$
A \neq \varlimsup\left(\left\{P_{n}: n \neq m\right\} \cup\left\{Q_{1}, Q_{2}\right\}\right) .
$$

Thus, $A$ is not maximal, a contradiction.

If $P \in L\left(s^{\prime}, s\right)$ is a nonzero projection orthogonal to all $P_{n}$, then, using similar arguments as above, we get that $\varlimsup\left(\left\{P_{n}\right\}_{n \in \mathbb{N}} \cup\{P\}\right)$ is a closed commutative ${ }^{*}$-subalgebra of $L\left(s^{\prime}, s\right)$ bigger than $A$ which leads to a contradiction.

(ii) $\Rightarrow$ (iii). Since $A$ is commutative thus $A \subset A^{\prime}$. Now, let us suppose that there is $x \in$ $A^{\prime} \backslash A$. By Proposition 4.9, $x^{*} \in A^{\prime}$ so $x+x^{*}, x-x^{*} \in A^{\prime}$, and, moreover, $x^{*} \notin A$. Since $x=\frac{1}{2}\left(x+x^{*}\right)+\frac{1}{2}\left(x-x^{*}\right)$ thus $x+x^{*} \notin A$ or $x-x^{*} \notin A$. Without loss of generality let us assume that $x+x^{*} \notin A$. Operator $x+x^{*}$ is self-adjoint hence it has the spectral representation of the form $\sum_{m=1}^{\infty} \mu_{m} Q_{m}$. Then, by Propositions 4.2 and 4.9, $Q_{m} \in A^{\prime}$ for all $m \in \mathbb{N}$ and there exists $m_{0}$ for which $Q_{m_{0}} \notin A$ (otherwise $x+x^{*} \in A$ ). Let $J:=\left\{n: P_{n} \preceq Q_{m_{0}}\right\}$ (see the definition of $\preceq$ in Lemma 4.4). Since $Q_{m_{0}}$ is finite dimensional thus $J$ is finite. One can easily check that $Q_{m_{0}}-\sum_{j \in J} P_{j}$ is a projection (if $J=\emptyset$, then $\sum_{j \in J} P_{j}:=0$ ). Moreover,

$$
\left(Q_{m_{0}}-\sum_{j \in J} P_{j}\right) P_{k}=0
$$

for all $k \in \mathbb{N}$. Indeed, if $k \in J$, then from the definition of the relation $\preceq, Q_{m_{0}} P_{k}=P_{k}$, so

$$
\left(Q_{m_{0}}-\sum_{j \in J} P_{j}\right) P_{k}=Q_{m_{0}} P_{k}-P_{k}=0
$$

Let $k \notin J$. We have $Q_{m_{0}} P_{k}=P_{k} Q_{m_{0}}$ because $Q_{m_{0}} \in A^{\prime}$. This implies that $Q_{m_{0}} P_{k}$ is a projection and $\operatorname{im} Q_{m_{0}} P_{k}=\operatorname{im} Q_{m_{0}} \cap \operatorname{im} P_{k}$. Therefore, since $P_{k}$ are one-dimensional thus $Q_{m_{0}} P_{k}=P_{k}$ or $Q_{m_{0}} P_{k}=0$. But, from the assumption, $Q_{m_{0}} P_{k} \neq P_{k}$ so $Q_{m_{0}} P_{k}=0$. Now,

$$
\left(Q_{m_{0}}-\sum_{j \in J} P_{j}\right) P_{k}=Q_{m_{0}} P_{k}=0 .
$$

Since the sequence $\left(P_{n}\right)_{n \in \mathbb{N}}$ is complete, (7) implies that $Q_{m_{0}}-\sum_{j \in J} P_{j}=0$. Hence $Q_{m_{0}} \in A$, a contradiction.

(iii) $\Rightarrow$ (i). Follows directly from the definition of the comutant of $A$.

Remark 4.11. (i) Since $\left(P_{n}\right)_{n \in \mathbb{N}}$ is a sequence of pairwise orthogonal one-dimensional projections so $P_{n}=\left\langle\cdot, e_{n}\right\rangle e_{n}$, where $\left(e_{n}\right)_{n \in \mathbb{N}} \subset s$ is an orthonormal system in $l_{2}$. Then $\lambda^{\infty}\left(\left\|P_{n}\right\|_{q}\right)=$ $\lambda^{\infty}\left(\left|e_{n}\right|_{q}\right)$ as Fréchet ${ }^{*}$-algebras. Indeed, from the Hölder inequality, if $\xi \in s, q \in \mathbb{N}_{0}$, then

$$
|\xi|_{q}^{2} \leq|\xi|_{l_{2}}|\xi|_{2 q}
$$

Hence

$$
1 \leq\left|e_{n}\right|_{q} \leq\left|e_{n}\right|_{q}^{2}=\left.|| P_{n}\right|_{q}=\left|e_{n}\right|_{q}^{2} \leq\left|e_{n}\right|_{2 q} .
$$

This implies that $\lambda^{\infty}\left(\| P_{n}||_{q}\right)=\lambda^{\infty}\left(\left|e_{n}\right|_{q}\right)$ as Fréchet spaces, and, since the algebra operations are the same in both algebras, $\lambda^{\infty}\left(\left\|P_{n}\right\|_{q}\right)=\lambda^{\infty}\left(\left|e_{n}\right|_{q}\right)$ as Fréchet ${ }^{*}$-algebras.

(ii) The sequence $\left(e_{n}\right)_{n \in \mathbb{N}}$ from the previous item need not to be an orthonormal basis of $l_{2}$. Indeed, let $\left(e_{n}\right)_{n \in \mathbb{N}}$ be an orthonormal basis of $l_{2}$ such that $e_{n} \in s$ for $n \in \mathbb{N} \backslash\{1\}$ and $e_{1} \notin s$. Clearly, $\left(e_{n}\right)_{n \in \mathbb{N} \backslash\{1\}}$ is not an orthonormal basis of $l_{2}$ and $\left(\left\langle\cdot, e_{n}\right\rangle e_{n}\right)_{n \in \mathbb{N} \backslash\{1\}}$ is a complete sequence in $L\left(s^{\prime}, s\right)$. 
(iii) By the Kuratowski-Zorn lemma, every closed commutative ${ }^{*}$-subalgebra of $L\left(s^{\prime}, s\right)$ is contained in some maximal commutative ${ }^{*}$-subalgebra of $L\left(s^{\prime} s\right)$. If $\left\{P_{n}\right\}_{n \in \mathbb{N}}$ is a sequence of pairwise orthogonal finite dimensional projections, then, by Proposition 4.6, $\operatorname{alg}\left(\left\{P_{n}\right\}_{n \in \mathbb{N}}\right)$ is a closed commutative ${ }^{*}$-subalgebra of $L\left(s^{\prime}, s\right)$ so it is contained in some maximal commutative ${ }^{*}$-subalgebra $\operatorname{alg}\left(\left\{Q_{n}\right\}_{n \in \mathbb{N}}\right)$ of $L\left(s^{\prime} s\right)$, where $\left\{Q_{n}\right\}_{n \in \mathbb{N}}$ is a complete sequence of one-dimensional projections in $L\left(s^{\prime}, s\right)$ (see Theorems 4.8 and 4.10). Now, applying Lemma 4.4 (i), it is easy to show that the sequence $\left\{P_{n}\right\}_{n \in \mathbb{N}}$ can be extended to some complete sequence of projections belonging to $L\left(s^{\prime}, s\right)$.

Corollary 4.12. Let $A$ be one of the following Fréchet ${ }^{*}$-algebras with the pointwise multiplication:

(i) the algebra $\mathcal{S}\left(\mathbb{R}^{n}\right)$ of rapidly decreasing smooth functions;

(ii) the algebra $\mathcal{D}(K)$ of test functions with support in a compact set $K \subset \mathbb{R}^{n}$, $\operatorname{int}(K) \neq \emptyset$;

(iii) the algebra $C_{a}^{\infty}(M)$ of smooth functions on a compact smooth manifold $M$ vanishing at $a \in M$;

(iv) the algebra $C_{a}^{\infty}(\bar{\Omega})$ of smooth functions on $\bar{\Omega}$ vanishing at $a \in \Omega$, where $\Omega \neq \emptyset$ is an open bounded subset of $\mathbb{R}^{n}$ with $C^{1}$-boundary;

(v) the algebra $\mathcal{E}_{a}(K)$ of Withney jets on a compact set $K \subset \mathbb{R}^{n}$ with the extension property, flat at $a \in K$ and such that $\operatorname{int}(K) \neq \emptyset$.

Then $A$ is isomorphic to $s$ as Fréchet space but it is not isomorphic to a closed commutative *-subalgebra of $L\left(s^{\prime}, s\right)$ as Fréchet ${ }^{*}$-algebra.

Proof. It is well known that spaces from items (i)-(v) are isomorphic to the space $s$ as Fréchet space (see e.g. [11, Ch. 31], [15, Satz 4.1]).

To prove the second assertion let us compare sets of *-multiplicative functionals. If $A$ is one of the spaces from items (i)-(v), then every point evaluation functional on $A$ is ${ }^{*}$-multiplicative and since the cardinal number of the underlying space is continuum, thus the cardinal number of the set of *-multiplicative functionals on $A$ is not less than continuum. On the other hand, by Corollary 4.5, the set of *-multiplicative functional on any infinite dimensional closed commutative ${ }^{*}$-subalgebra of $L\left(s^{\prime}, s\right)$ is at most countable, hence no of the spaces from items (i)-(v) is isomorphic to $A$.

It is clear that the algebra $s$ with the pointwise multiplication is a ${ }^{*}$-subalgebra of $L\left(s^{\prime}, s\right)$ (consider, for example, diagonals operators). The previous corollary shows that it is not the case for many other interesting Fréchet ${ }^{*}$-algebras isomorphic to $s$ (as Fréchet spaces). This leads to the following:

Open Problem 4.13. Does every closed commutative ${ }^{*}$-subalgebra of $L\left(s^{\prime}, s\right)$ is isomorphic to some closed ${ }^{*}$-subalgebra of the algebra $s$ with the pointwise multiplication?

We say that an orthonormal system $\left(e_{n}\right)_{n \in \mathbb{N}}$ in $l_{2}$ is $s$-complete, if every $e_{n}$ belongs to $s$ and for every $\xi \in s$ the following implication holds: if $\left\langle\xi, e_{n}\right\rangle=0$ for every $n \in \mathbb{N}$, then $\xi=0$. One can easily show that an orthonormal system $\left(e_{n}\right)_{n \in \mathbb{N}}$ is $s$-complete if and only if the sequence of projections $\left(\left\langle\cdot, e_{n}\right\rangle e_{n}\right)_{n \in \mathbb{N}}$ is complete in $L\left(s^{\prime}, s\right)$. Hence, by Theorem 4.10 and Remark 4.11 , Problem 4.13 is equivalent to the following.

Open Problem 4.14. Let $\left(e_{n}\right)_{n \in \mathbb{N}}$ be s-complete, orthonormal system in $l_{2}$. Is the algebra $\lambda^{\infty}\left(\left|e_{n}\right|_{q}\right)$ isomorphic to some closed ${ }^{*}$-subalgebra of the algebra $s$ ? 


\section{$5 \quad$ Functional calculus in $L\left(s^{\prime}, s\right)$}

If $x$ is a normal operator in $L\left(s^{\prime}, s\right) \subset K\left(l_{2}\right)$ and $f$ is a continuous function on the spectrum $\sigma(x)$ of $x$ vanishing at zero, then, from the continuous functional calculus for normal operators (see e.g. [11, Prop. 17.20]), $f(x)$ is the uniquely determined operator in $K\left(l_{2}\right)$. In this section, we want to describe these functions $f$ for which $f(x)$ is again in $L\left(s^{\prime}, s\right)$.

From the general theory of Fréchet $m$-locally convex algebras we get the holomorphic functional calculus on $L\left(s^{\prime}, s\right)$ (see Prop. 2.5 and [12, Lemma 1.3], [17, Th. 12.16]). Precisely, if $x$ is an arbitrary operator in $L(s, s)$ and $f$ is a holomorphic function on an open neighborhood $U$ of $\sigma(x)$ with $f(0)=0$, then $f(x) \in L\left(s^{\prime}, s\right)$, and, moreover, the map $\Phi: H_{0}(U) \rightarrow L\left(s^{\prime}, s\right)$, $f \mapsto f(x)$ is a continuous homomorphism $\left(H_{0}(U)\right.$ stands for the space of holomorphic functions vanishing at zero).

Let us recall that $f: X \rightarrow \mathbb{C}(X \subset \mathbb{C}, 0 \in X)$ is a Hölder continuous at zero function if there is $\theta \in(0,1]$ and $C>0$ such that $|f(t)-f(0)| \leq C|t|^{\theta}$ for all $t$ in the neighborhood of 0 . As a consequence of Theorem 3.1 we get the following Hölder continuous functional calculus for normal operators in $L\left(s^{\prime}, s\right)$.

Corollary 5.1. If $x \in L\left(s^{\prime}, s\right) \subset K\left(l_{2}\right)$ is normal, then for every Hölder continuous at zero function $f: \sigma(x) \rightarrow \mathbb{C}$ with $f(0)=0$, the operator $f(x) \in L\left(s^{\prime}, s\right)$ as well. In particular, for every normal operator $x \in L\left(s^{\prime}, s\right)$ with $\sigma(x) \subset[0, \infty)$ and $\theta \in(0, \infty), x^{\theta} \in L\left(s^{\prime}, s\right)$.

Proof. Let $x=\sum_{n \in \mathcal{N}} \lambda_{n} P_{n}$ be a normal operator in $L\left(s^{\prime}, s\right)$ with the nonnegative spectrum and let $\theta \in(0, \infty)$. If $\theta \in(0,1]$, then, by Theorem 3.1, $x^{\theta}=\sum_{n=1}^{\infty} \lambda_{n}^{\theta} P_{n} \in L\left(s^{\prime}, s\right)$ and for $\theta \in(1, \infty)$ we have $x^{\theta}=x^{\lfloor\theta\rfloor} \cdot x^{\theta-\lfloor\theta\rfloor} \in L\left(s^{\prime}, s\right)$, where $\lfloor\theta\rfloor$ is the floor of $\theta$.

Now, let $x=\sum_{n \in \mathcal{N}} \lambda_{n} P_{n} \in L\left(s^{\prime}, s\right)$ be normal and let $f: \sigma(x) \rightarrow \mathbb{C}$ be a Hölder continuous at zero function with $f(0)=0$. Then $|f| \leq C|\cdot|^{\theta}$ for some $C>0$ and $\theta \in(0,1]$. Hence $\sum_{n \in \mathcal{N}}\left\|f\left(\lambda_{n}\right) P_{n}\right\|_{q} \leq C \sum_{n \in \mathcal{N}}\left|\lambda_{n}\right|^{\theta}\left\|P_{n}\right\|_{q}<\infty$ so, by Corollary 3.6, $f(x) \in L\left(s^{\prime}, s\right)$.

For a normal operator $x$ in $L\left(s^{\prime}, s\right)$ with the spectral representation $x=\sum_{n=1}^{\infty} \lambda_{n} P_{n}$, we define the function space

$$
C_{s}(\sigma(x)):=\left\{f: \sigma(x) \longrightarrow \mathbb{C}: f(0)=0,\left(f\left(\lambda_{n}\right)\right)_{n \in \mathbb{N}} \in \lambda^{\infty}\left(\left\|P_{n}\right\|_{q}\right)\right\} .
$$

It is easy to show that the space $C_{s}(\sigma(x))$ with a system $\left(c_{q}\right)_{q \in \mathbb{N}_{0}}, c_{q}(f):=\sup \left|f\left(\lambda_{n}\right)\right|\left\|P_{n}\right\|_{q}$ of seminorms, pointwise multiplication and conjugation is a Fréchet ${ }^{*}$-algebra.

Theorem 5.2. If $x=\sum_{n=1}^{\infty} \lambda_{n} P_{n}$ is an infinite dimensional normal operator in $L\left(s^{\prime}, s\right)$, then the map

$$
\Phi: C_{s}(\sigma(x)) \longrightarrow \operatorname{alg}(x), \quad \Phi(f):=f(x):=\sum_{n=1}^{\infty} f\left(\lambda_{n}\right) P_{n}
$$

is a Fréchet algebra isomorphism such that $\Phi(\mathrm{id})=x$ and $\Phi(\bar{f})=\Phi(f)^{*}$.

Proof. By Theorem 4.8, $\Phi$ is well defined, and, of course, $\Phi(\mathrm{id})=x, \Phi(\bar{f})=\Phi(f)^{*}$. The space $\operatorname{alg}(x)$ is a nuclear Fréchet space (as a closed subspace of nuclear Fréchet space $L\left(s^{\prime}, s\right)$ ) so $\lambda^{\infty}\left(\left\|P_{n}\right\|_{q}\right) \cong \operatorname{alg}(x)$ (see Theorem 4.8) is a nuclear Fréchet space as well. Thus, by the Grothendieck-Pietsch theorem (see e.g. [11, Th. 28.15]), for given $q \in \mathbb{N}_{0}$ one can find $r \in \mathbb{N}_{0}$ 
such that $C:=\sum_{n=1}^{\infty} \frac{\left\|P_{n}\right\|_{q}}{\left\|P_{n}\right\|_{r}}<\infty$. Hence

$$
\begin{aligned}
\|\Phi(f)\|_{q} & \leq \sum_{n=1}^{\infty}\left|f\left(\lambda_{n}\right)\|\| P_{n}\left\|_{q}=\sum_{n=1}^{\infty} \mid f\left(\lambda_{n}\right)\right\|\left\|P_{n}\right\|_{r} \frac{\left\|P_{n}\right\|_{q}}{\left\|P_{n}\right\|_{r}}\right. \\
& \leq \sup _{n \in \mathbb{N}}\left|f\left(\lambda_{n}\right)\left\|\mid P_{n}\right\|_{r} \cdot \sum_{n=1}^{\infty} \frac{\left\|P_{n}\right\|_{q}}{\left\|P_{n}\right\|_{r}}=C c_{r}(f)\right.
\end{aligned}
$$

thus $\Phi$ is continuous.

Clearly, $\Phi$ is injective. To prove that $\Phi$ is also surjective, let us take $y \in \operatorname{alg}(x)$. Since, by Theorem 4.8, $\left(P_{n}\right)_{n \in \mathbb{N}}$ is a Schauder basis, so there is a sequence $\left(\mu_{n}\right)_{n \in \mathbb{N}}$ such that $y=$ $\sum_{n=1}^{\infty} \mu_{n} P_{n}$. Let $g\left(\lambda_{n}\right):=\mu_{n}$ for $n \in \mathbb{N}$. Then

$$
\sup _{n \in \mathbb{N}}\left|g\left(\lambda_{n}\right)\right||| P_{n}\left\|_{q}=\sup _{n \in \mathbb{N}}\left|\mu_{n}\right|\right\| P_{n} \|_{q}<\infty
$$

hence $g \in C_{s}(\sigma(x))$ and of course $\Phi(g)=y$.

Acknowledgements. The author is very indebted to Paweł Domański and Krzysztof Piszczek for several helpful conversations and especially for discussions on their papers [6], [13].

\section{References}

[1] S. J. Bhatt, A. Inoue, H. Ogi, Spectral invariance, $K$-theory isomorphism and an application to the differential structure of $C^{*}$-algebras. J. Operator Theory 49 (2003), no. 2, 389-405.

[2] B. Blackadar, J. Cuntz, Differential Banach algebra norms and smooth subalgebras of $C^{*}$-algebras. J. Operator Theory 26 (1991), no. 2, 255-282.

[3] A. Connes, Noncommutative geometry. Academic Press, Inc., San Diego, CA, 1994.

[4] J. B. Conway, A course in functional analysis. Second edition. Graduate Texts in Mathematics, 96. Springer-Verlag, New York, 1990.

[5] J. Cuntz, Bivariante K-Theorie für lokalkonvexe Algebren und der Chern-Connes-Charakter. Doc. Math. 2 (1997), 139-182.

[6] P. Domański, Algebra of smooth operators. Unpublished note.

[7] G. A. Elliot, T. Natsume, R. Nest, Cyclic cohomology for one-parameter smooth crossed products. Act. Math. 160 (1998), 285-305.

[8] M. Fragoulopoulou, Topological algebras with involution. North-Holland Mathematics Studies, 200. Elsevier Science B.V., Amsterdam, 2005.

[9] H. Glöckner, B. Langkamp, Topological algebras of rapidly decreasing matrices and generalizations. Topology Appl. 159 (2012), no. 9, 2420-2422.

[10] G. Köthe, Topological vector spaces II. Springer-Verlag, Berlin-Heidelberg-New York 1979.

[11] R. Meise, D. Vogt, Introduction to functional analysis. Oxford University Press, New York 1997.

[12] N. C. Phillips, K-theory for Fréchet algebras. Internat. J. Math. 2 (1991), no. 1, 77-129.

[13] K. Piszczek, On a property of PLS-spaces inherited by their tensor products. Bull. Belg. Math. Soc. Simon Stevin 17 (2010), 155-170.

[14] L. B. Schweitzer, $A$ short proof that $M_{n}(A)$ is local if $A$ is local and Fréchet. Internat. J. Math. 3 (1992), no. 4, 581-589. 
[15] D. Vogt, Ein Isomorphiesatz für Potenzreihenräume. Arch. Math. (Basel) 38 (1982), no. 6, 540-548.

[16] D. Vogt, On the functors $\operatorname{Ext}^{1}(E, F)$ for Fréchet spaces. Studia Math. 85 (1987), no. 2, 163-197.

[17] W. Żelazko, Selected topics in topological algebras. Lectures 1969/1970, Lectures Notes Series, No. 31. Matematisk Institut, Aarhus Universitet, Aarhus 1971.

T. Ciaś

Faculty of Mathematics and Comp. Sci.

A. Mickiewicz University in Poznań

Umultowska 87

61-614 Poznań, POLAND

e-mail: tcias@amu.edu.pl 